\title{
Nonsymmetrical Segregation of Solutes in Periodic Misfit Dislocations Separated Tilt Grain Boundaries
}

\section{Supplementary Information}

Hongbo Xie ${ }^{\dagger}$, Qiuyan Huang ${ }^{\star} *$, Junyuan $\mathrm{Bai}^{\dagger}$, Shanshan Li†, Yang Liu ${ }^{\ddagger}$, Jianguang Feng ${ }^{\ddagger}$, Yuansheng Yang ${ }^{\ddagger}$, Hucheng Pan ${ }^{\dagger *}$, Hongxiao Li ${ }^{\S}$, Yuping Ren ${ }^{\S}$, , Gaowu Qin ${ }^{\S}, \#$

${ }^{\dagger}$ Key Laboratory for Anisotropy and Texture of Materials (Ministry of Education), School of Materials Science and Engineering, Northeastern University, Shenyang 110819, China

\#Institute of Metal Research, Chinese Academy of Sciences, Shenyang 110016, China

\$ State Key Laboratory of Rolling and Automation, Northeastern University, Shenyang 110819, China

\# Research Center for Metal Wires, Northeastern University, Shenyang 110819, China

* Corresponding author E-mail: panhc@atm.neu.edu.cn (H. Pan); qyhuang16b@imr.ac.cn (Q. Huang). 


\section{Materials and Methods}

\subsection{Experimental procedures}

The alloy with nominal composition of $\mathrm{Mg}-2 \mathrm{Nd}-1 \mathrm{Mn}$ (wt.\%) was prepared by melting the pure $\mathrm{Mg}$ (99.9 wt.\%), Mg-5Mn (wt.\%) master alloy, and Mg-30Nd (wt.\%) master alloy in the induction furnace with protection of the argon atmosphere. The molten alloy was stirred and kept at $760{ }^{\circ} \mathrm{C}$ for 5 minutes and poured into a steel mold preheated to $300{ }^{\circ} \mathrm{C}$. The as-cast sample was solution treated at $520{ }^{\circ} \mathrm{C}$ for $12 \mathrm{~h}$, followed by water quenching. The solution treated alloy was machined into a set of standard compressed samples $\left(\Phi 10 \times 15 \mathrm{~mm}^{3}\right)$ and then compressed by $25 \%$ $(15 \mathrm{~mm} \rightarrow 11.25 \mathrm{~mm})$ at room temperature, with a constant strain rate of $1 \times 10^{-3} \mathrm{~s}^{-1}$. The annealing heat treatment of the compressed samples was performed at $250^{\circ} \mathrm{C}$ for $1 \mathrm{~h}$.

The TEM specimens with a diameter of $3 \mathrm{~mm}$ were prepared by twin jet electro-polishing at $40{ }^{\circ} \mathrm{C}$ in mixture solution of $5.3 \mathrm{~g}$ lithium chloride, $11.2 \mathrm{~g}$ magnesium perchlorate, $500 \mathrm{ml}$ methanol and $100 \mathrm{ml}$ 2-butoxy ethanol, and subsequently ion milling with low energy electron beam. Finally, Gatan SOLARUS (950) Plasma Cleaning System was used to clean up the sample surfaces. TEM and STEM observations were carried out by using the JEM-ARM200F at an accelerating voltage of $200 \mathrm{kV}$, equipped with probe Cs corrector and cold field emission gun. The probe convergence is $25 \mathrm{mrad}$ which yields a probe size of less than $0.1 \mathrm{~nm}$, and the camera length was set to $8 \mathrm{~cm}$ which yields a collection semi-angle of 48-327 mrad. STEM-EDS mappings were acquired by scanning the beam near the GBs, using the NSS3 spectral analysis software developed by Thermo Fisher Scientific Inc. The EDS collection solid angle is about $0.8 \mathrm{sr}$. The probe size was $1.2 \AA$ with a probe current of about $60 \mathrm{pA}$.

\subsection{Computational methods}

For numerical implementation of the molecular dynamics (MD) simulations, we used the large-scale atomic/molecular massively parallel simulator (LAMMPS) software ${ }^{1}$. The selection of interatomic potential is a key factor determining the accuracy of MD simulation results. Therefore, the interaction of $\mathrm{Mg}-\mathrm{Mg}$ is described by embedded-atom method (EAM) potential ${ }^{2}$. The stable grain boundaries are formed at $50 \mathrm{~K}$ temperature using Nose-Hoover algorithm and NPT ensemble is used to relieve the external stress, and then a quenching process was applied to minimize the total potential energy of the whole system.

An open visualization tool (OVITO software package) was used for Voronoi analysis of each relaxed tilt grain boundaries. The atomic volume of a perfect crystal of $\mathrm{Mg}$ is $22.8629 \AA^{3}$, and a 
scale range of $-8.15 \%$ to $+9.35 \%$ was applied to the Voronoi analysis map.

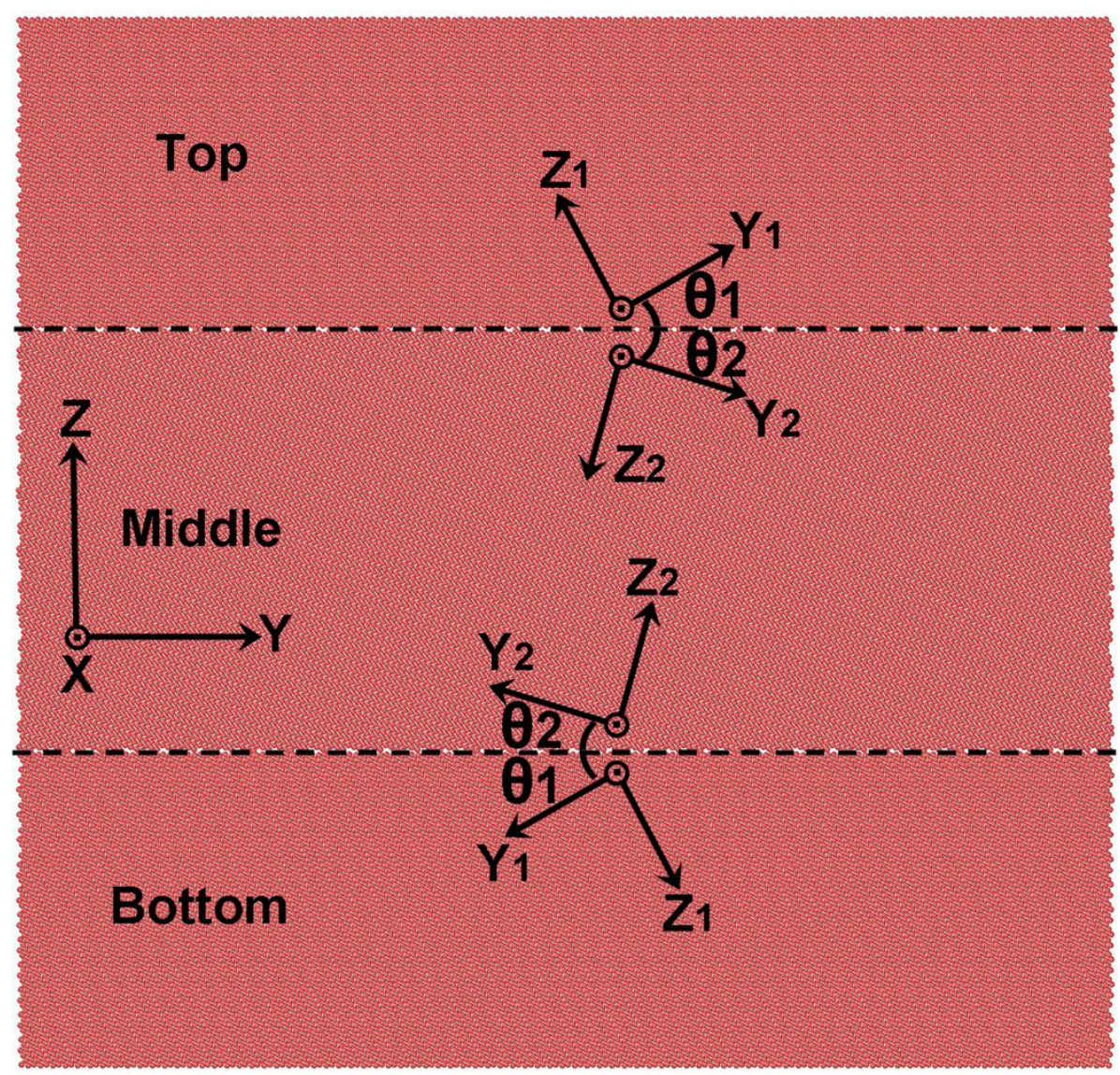

Figure S1. Molecular dynamics simulation cell. The simulation cell consists of two tilted grain boundaries, which divides the model into three parts, i.e., the top, the middle, and the bottom ones. The $\mathrm{X}$ axis is $[11 \overline{2} 0]$ as the tilt axis, and the $\mathrm{Z}$ axis is the normal of GB plane (the dash lines). The middle part rotates $\theta_{2}$ clockwise, the top one and the bottom one rotates $\theta_{1}$ counterclockwise about $X$ axis. The crystal orientation of $Y_{1}, Y_{2}$ and $Z_{1}, Z_{2}$ are $[10 \overline{1}$ $0]$ and [0001], respectively. Periodic boundary conditions (PBCs) are applied in the $\mathrm{X}, \mathrm{Y}$ and $\mathrm{Z}$ directions. The lengths of $\mathrm{X}, \mathrm{Y}$ and $\mathrm{Z}$ dimensions are $12.5 \AA, 533 \AA$, and $570 \AA$, respectively.

\section{References}

(1) Plimpton, S.J. Fast Parallel Algorithms for Short-Range Molecular Dynamics. J. Comp. Phys. 1995, 117, 1-19.

(2) Liu, X. Y. Adams, J. B. Ercolessi, F. Moriarty, J. EAM potential for magnesium from quantum mechanical forces. Modelling Simul. Mater. Sci. Eng. 1996, 4, 293-303. 


\section{Supplementary Figures}

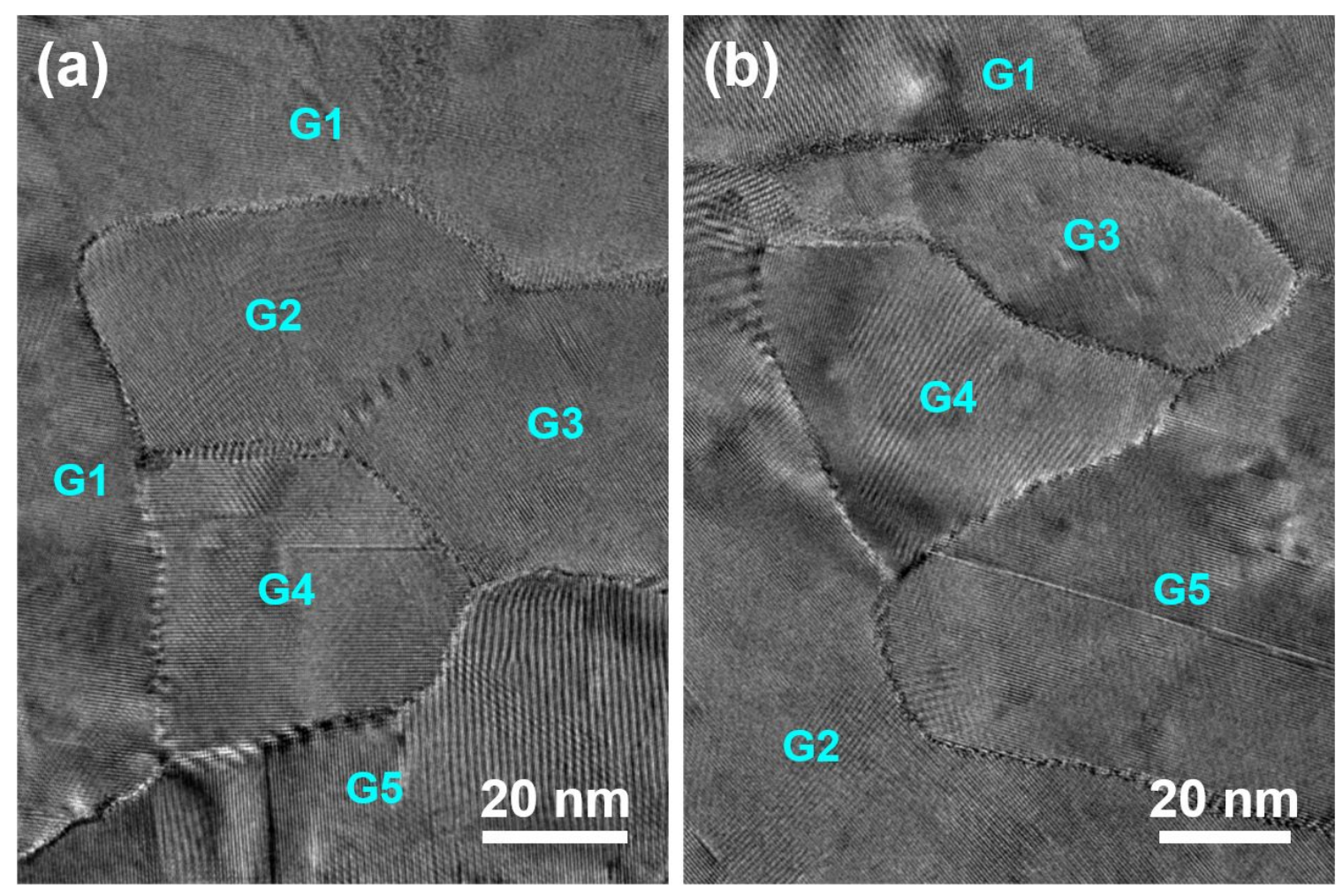

Figure S2. High-resolution TEM images showing micro/nano-scale polycrystalline with their $\langle 11 \overline{2} 0\rangle$ directions parallel to each other in a compressed and annealed Mg-2Nd-1Mn sample. The electron beam is parallel to $<11 \overline{2} 0>$ direction. 


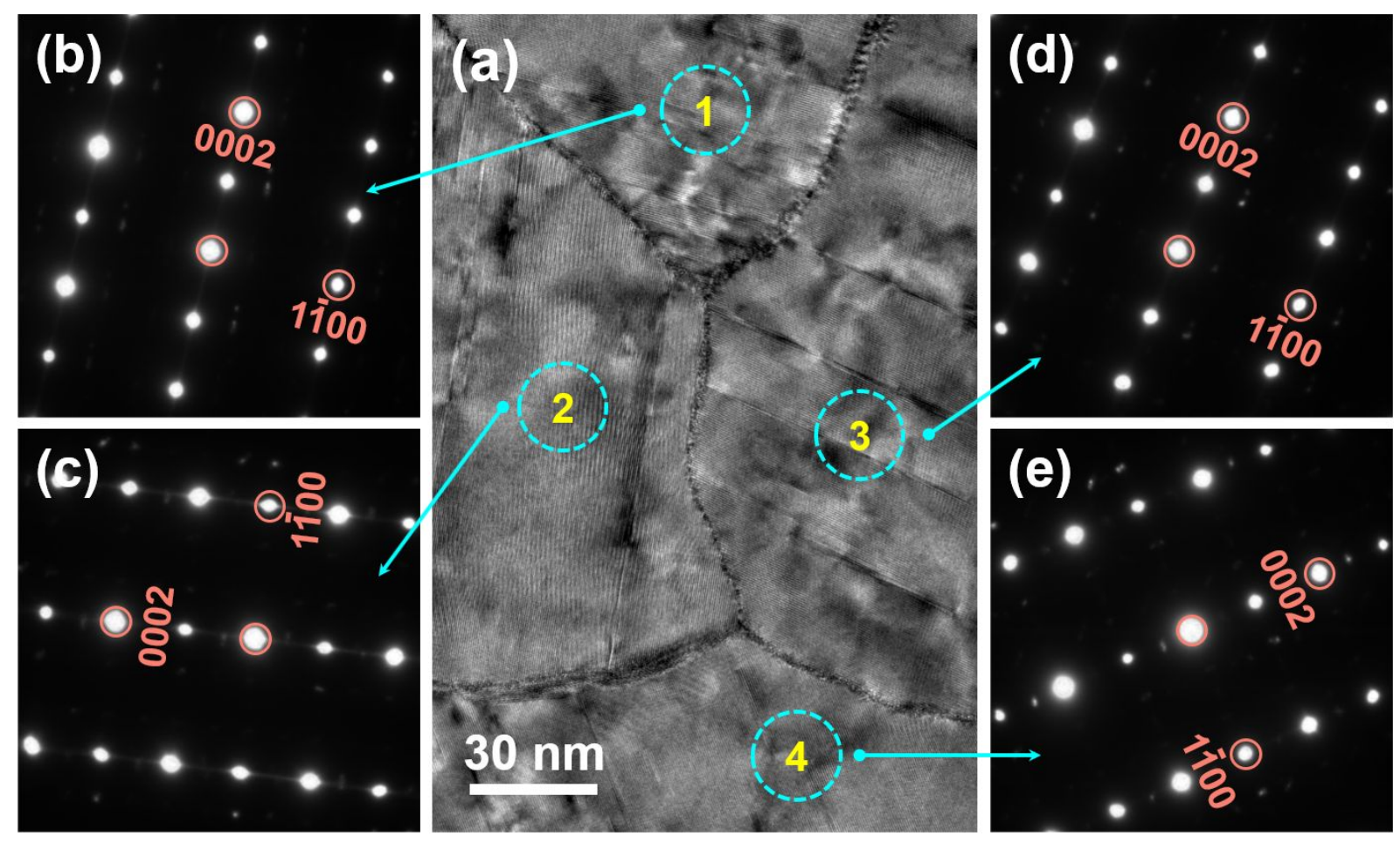

Figure S3. Bright-field image (a) and the corresponding select-area electron diffraction (SAED) patterns (b-e) showing the four grains with their $<11 \overline{2} 0>$ directions parallel to each other in a compressed and annealed Mg-2Nd-1Mn sample. The electron beam is parallel to $<11 \overline{2} 0>$ direction. 

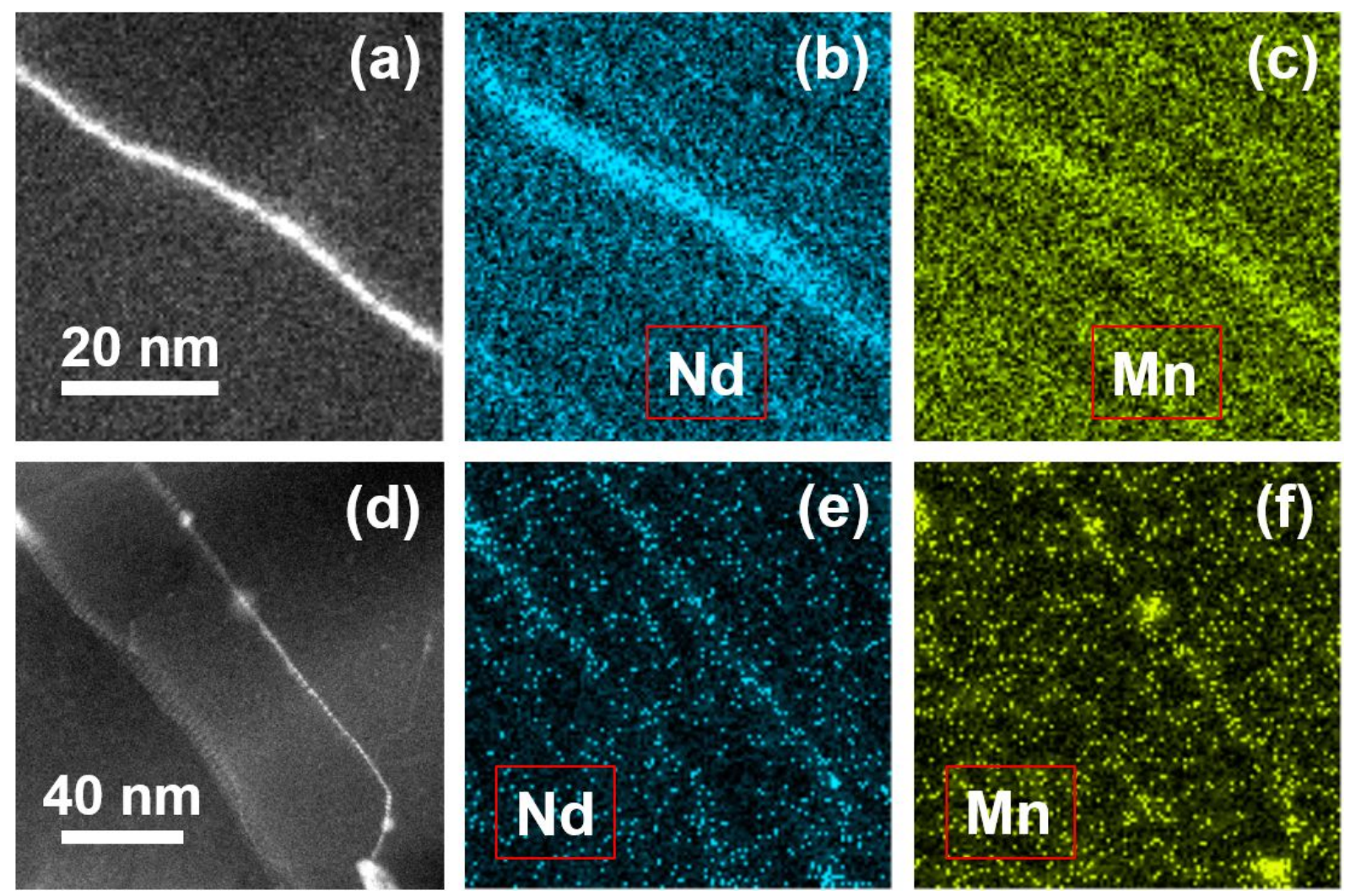

Figure S4. STEM-EDS mapping results of the Mg-2Nd-1Mn alloy compressed by $25 \%$ and annealed for $1 \mathrm{~h}$ at 250 ${ }^{\circ} \mathrm{C}$. HAADF-STEM images (a and d) and the corresponding EDS mapping images related to the elements $\mathrm{Nd}(\mathrm{b}$ and e) and $\mathrm{Mn}$ (c and f). The electron beam is parallel to $<11 \overline{2} 0>$ direction. 
Table S1. Interfacial parameters of the four tilt grain boundaries.

\begin{tabular}{|c|c|c|c|c|c|c|c|c|c|c|}
\hline \multirow{3}{*}{ Types } & \multicolumn{6}{|c|}{ Lattice parameters } & \multicolumn{4}{|c|}{ Grain boundary characteristics } \\
\hline & \multicolumn{3}{|c|}{ Grain 1} & \multicolumn{3}{|c|}{ Grain 2} & \multicolumn{2}{|c|}{ Grain 1} & \multicolumn{2}{|c|}{ Grain 2} \\
\hline & $a(\AA)$ & $c(\AA)$ & Axial ratio $(c / a)$ & $a(\AA)$ & $c(\AA)$ & Axial ratio $(c / a)$ & Interfaces & Tilt angles $\left({ }^{\circ}\right)$ & Interfaces & Tilt angles $\left({ }^{\circ}\right)$ \\
\hline I (ATGB) & 3.15 & 5.44 & 1.73 & 3.31 & 5.17 & 1.56 & $(30 \overline{3} 5)$ & $\sim 51$ & $(40 \overline{4} 1)$ & $\sim 79$ \\
\hline II (ATGB) & 3.15 & 5.44 & 1.73 & 3.20 & 5.39 & 1.68 & $(30 \overline{3} 3)$ & $\sim 116$ & $(10 \overline{1} 6)$ & $\sim 17$ \\
\hline III (STGB) & 3.20 & 5.18 & 1.62 & 3.23 & 5.21 & 1.61 & $(10 \overline{1} 5)$ & $\sim 20.5$ & $(10 \overline{1} 5)$ & $\sim 20$ \\
\hline IV (STGB) & 3.14 & 5.23 & 1.67 & 3.22 & 5.15 & 1.60 & $(20 \overline{2} 3)$ & 49 & $(20 \overline{2} 3)$ & 51 \\
\hline
\end{tabular}


Acknowledgements

The authors acknowledge the National Key Research and Development Program of China (Nos. 2016YFB0701202, 2016YFB0301105, and 2016YFB0701200), the National Natural Science Foundation of China (Nos. 51525101, 51971053, U1610253, and 51701211), the Project of Promoting Talents in Liaoning Province (No. XLYC1808038), and the Project funded by China Postdoctoral Science Foundation (2020M670774). We thank Prof. Yonghui Sun and Dr. Yu Dong (Analytical and Testing Center, Northeastern University) for their help with the Cs-corrected HAADF-STEM technique. We are grateful to Prof. Kui Du (Institute of Metal Research, Chinese Academy of Sciences) for his help in the data collection of atomic coordinates. The authors extend their gratitude to Prof. Jianfeng Jin and Dr. Peijun Yang (Northeastern University) for their contributions on molecular dynamics simulations. 